\title{
Insegurança alimentar e fatores associados em beneficiários de programas sociais de combate à fome no município de Petrópolis-RJ, Brasil
}

\section{Food insecurity and associated factors among public policies beneficiaries in the municipality of Petropolis-RJ, Brazil}

\author{
Aline Gaudard e Silva, 2 \\ Cintia Chaves Curionil,3 \\ ${ }^{1}$ Curso de Nutrição. Faculdade Arthur Sá Earp \\ Neto. Petrópolis, RJ, Brasil. \\ ${ }^{2}$ Departamento de Epidemiologia. Instituto de \\ Medicina Social. Universidade do Estado do Rio \\ de Janeiro. Rio de Janeiro, RJ, Brasil. \\ ${ }^{3}$ Departamento de Nutrição Social. Instituto \\ de Nutrição. Universidade do Estado do Rio de \\ Janeiro. Rio de Janeiro, RJ, Brasil. \\ Correspondência / Correspondence \\ Aline Gaudard e Silva \\ E-mail: alinegaudard@yahoo.com.br
}

\section{Resumo}

A produção de alimentos no Brasil é suficiente para toda sua população. No entanto, milhões de brasileiros continuam sofrendo por fome. Foram lançados pelo governo programas inovadores para combater esse quadro. Este estudo seccional teve como objetivo investigar a prevalência de insegurança alimentar (IA) entre os beneficiários de dois programas locais de combate à fome: abastecimento de alimentos (CCFF) e restaurante popular (RP), bem como estabelecer a associação entre IA e alguns marcadores de desigualdade social. No município de Petrópolis-RJ, 195 famílias beneficiárias desses programas foram avaliadas quanto à situação de IA através da Escala Brasileira de Insegurança Alimentar (EBIA). Variáveis socioeconômicas e demográficas também foram avaliadas. A prevalência de IA foi de $42,9 \%$ nas famílias beneficiárias do RP e 72,2\% nas famílias do CCFF. O número de moradores no domicílio, presença de moradores menores de 20 anos de idade, presença de moradores menores de 6 anos, classe socioeconômica, renda familiar mensal per capita, cor/raça do chefe da família, posse, tipo de construção e número de cômodos do domicílio foram significativamente associados com IA ( $p<0,05)$. Garantir a segurança alimentar exige ações coordenadas e articuladas entre as políticas estruturais e as emergenciais, não sendo tarefa fácil, especialmente no Brasil, historicamente marcado pela desigualdade social. Os resultados aqui apresentados são fundamentais para identificar as famílias mais vulneráveis a serem priorizadas. O primeiro passo foi dado, mas ainda há muito a ser feito. Ferramentas simples, como a EBIA, podem ser utilizadas para monitorar e combater a desigualdade.

Palavras-chave: Segurança Alimentar. Desigualdade Social. Programas Nutricionais. 


\section{Abstract}

Brazil produces enough food to feed its population. However, millions of Brazilians continue to suffer from hunger. To combat poverty and hunger, innovative programs have been launched by the government. This cross-sectional study aimed to investigate the prevalence of food insecurity (FI) among beneficiaries of two local programs to combat hunger: food supply (FS) and popular restaurant (PR) as well as to establish the association between food insecurity and some markers of social inequality. In the municipality of Petropolis-RJ, Brazil, 195 beneficiary families of these programs were assessed to investigate the FI by the Brazilian Food Insecurity Scale (EBIA). Socioeconomic and demographic variables were also evaluated. The prevalence of FI was $42.9 \%$ in the families beneficiaries of PR and $72.2 \%$ in the families included in the FS program. The number of family members, presence of family members under 20 and 6 years, economic level, family monthly per capita income, head of family race, home ownership, type of construction and number of rooms of the household were significantly associated with food insecurity $(\mathrm{p}<0.05)$. Ensuring food security demands crucial coordinated actions involving the appropriate linkage between the structural policies and the emergency interventions, it is not an easy task, especially in Brazil, historically marked by social inequality. The results were important to identify most vulnerable families that should be prioritized for assistance measures. The first step has been taken, but much more remains to be done. Simple tools such as EBIA can be used to monitor and combat inequality.

Key words: Food Security. Social Inequity. Public policies. Nutrition Programs.

\section{Introdução}

De acordo com o Conselho Nacional de Segurança Alimentar e Nutricional, a segurança alimentar e nutricional (SAN) consiste no direito de todos os cidadãos ao acesso regular e permanente a alimentos de qualidade, em quantidade suficiente, sem comprometer o acesso a outras necessidades essenciais, com base na promoção da saúde, respeitando as práticas alimentares, a diversidade cultural e a sustentabilidade ambiental, econômica e social. ${ }^{1}$ 
Apesar de o Brasil ser $100 \%$ autossuficiente em alimentos, dados do IBGE mostram que $39,8 \%$ dos seus cidadãos, o equivalente a aproximadamente 72 milhões de pessoas, vivem em estado de insegurança alimentar (IA). ${ }^{2}$ A Organização para a Alimentação e Agricultura das Nações Unidas (FAO) estima que o Brasil tem alimento suficiente para fornecer até 3,113 kcal/ dia para cada habitante, bem acima do mínimo recomendado de 1,850 kcal/dia. No entanto, observa-se que $6 \%$ dos brasileiros (11,9 milhões) têm consumo calórico continuamente abaixo desta recomendação mínima. ${ }^{3}$

O acesso aos alimentos é o principal fator determinante da IA no Brasil. A SAN é comprometida quando o acesso aos alimentos é irregular ou insuficiente, ou em casos em que ele é custoso e compromete grande parte da renda total familiar, prejudicando a satisfação de outras necessidades básicas de uma vida digna. A ampliação do acesso aos alimentos deve ser realizada através do aumento do poder aquisitivo da população e da redução dos custos da alimentação. No entanto, esses dois mecanismos não excluem a necessidade de programas emergenciais para combater a fome..$^{4,5}$

O programa "Fome Zero" foi criado em 2003 com o objetivo principal de erradicar a fome, através da mobilização de recursos políticos, financeiros, técnicos e outros. Recentemente, foi substituído pelo programa "Brasil sem Miséria". O "Fome Zero" combinava políticas estruturais - visando atacar as causas mais profundas da fome e da pobreza, políticas específicas - atendendo às necessidades das famílias em relação a situações agudas de fome e miséria, e políticas locais realizadas pelos governos estaduais e municipais com base em necessidades locais. ${ }^{6}$

Dentre as iniciativas para o barateamento das refeições, os restaurantes populares e os canais alternativos de comercialização têm mostrado boa resolutividade. Os restaurantes populares oferecem refeições prontas, de qualidade, a baixo custo, e são direcionados ao público de baixo poder aquisitivo que vive no meio urbano, com o objetivo de complementar ou suprir parte das necessidades nutricionais diárias. Os canais alternativos de comercialização, como, sacolões, varejões, feiras do produtor, buscam fornecer alimentos de qualidade e de baixo custo à população que não dispõe de meios permanentes para comprar alimentos na rede privada. ${ }^{4,7}$ Ambas as ações contribuem para o aumento da produção e distribuição de alimentos por pequenas e médias empresas, gerando emprego e renda. Além disso, também promovem hábitos alimentares saudáveis e fortalecimento da cidadania e do direito humano à alimentação adequada., 1,8,9

O presente estudo teve como objetivo investigar a prevalência de IA entre os beneficiários dos dois programas locais: abastecimento de alimentos e restaurante popular, bem como estabelecer a associação entre a IA e alguns marcadores de desigualdade social. 


\section{Metodologia}

Foi realizado estudo seccional, entre julho e outubro de 2009, com 195 famílias beneficiárias de dois programas locais de combate à fome no município de Petrópolis-RJ, Brasil. A amostra de conveniência foi composta por 90 famílias incluídas em um programa de fornecimento de alimentos - "Cesta Cheia, Família Feliz" (CCFF) -, residentes na área de cobertura de uma unidade da Estratégia de Saúde da Família, e 105 beneficiários do único Restaurante Popular (RP) do município. Foi entrevistado um representante de cada família, com idade igual ou maior que 18 anos, reconhecidamente conhecedor da dinâmica alimentar familiar.

Foi utilizado um questionário padronizado com perguntas relacionadas à segurança alimentar nos três últimos meses e às condições socioeconômicas da família. A IA foi avaliada pela Escala Brasileira de Insegurança Alimentar (EBIA), uma versão adaptada da escala proposta pelo Departamento de Agricultura dos Estados Unidos, ${ }^{10}$ validada para aplicação em área urbana no Brasil $^{11}$. O instrumento consiste em 15 perguntas direcionadas a um membro da família, com o objetivo de captar, através das respostas, as diferentes dimensões da (in)segurança alimentar familiar.

Dentre os 15 itens do instrumento, nove são relativos a adultos residentes no domicílio e seis são relativos a crianças. Em cada questão da escala, referindo-se aos últimos três meses, são atribuídas as opções de resposta "sim" e "não". Se a resposta for afirmativa, a frequência de ocorrência do evento no período é verificada, com as seguintes possibilidades de resposta: "quase todos os dias", "alguns dias" e "apenas um ou dois dias". A família foi então classificada em diferentes níveis de segurança alimentar - segurança alimentar, IA leve (receio ou medo de sofrer insegurança alimentar no futuro próximo), IA moderada (restrição na quantidade de alimentos na família) ou IA grave (fome entre pessoas adultas e/ou crianças da família), conforme o somatório das respostas positivas. ${ }^{11}$

Também foram mensuradas variáveis socioeconômicas e demográficas, incluindo composição familiar, classe econômica, renda, cor/raça, emprego, escolaridade e estado civil (do chefe de família), condições de habitação e saneamento básico, uso de supermercados ou outros recursos para compras de alimentos e participação em outros programas sociais do governo.

A classe econômica, de acordo com o Critério-Brasil (Associação Brasileira de Pesquisa de Mercado - ABEP), foi classificada como A, B, C, D e E, em ordem decrescente de acordo com o poder de compra. Isso equivale a uma renda mensal média de US\$ 1.600 para a classe A, US\$ 700 para a classe B, US\$ 290 para a classe C, US\$ 150 para classe D e US\$ 80 para classe E. ${ }^{12}$ 
A prevalência dos diferentes níveis de (in)segurança alimentar foi calculada em subgrupos, segundo as variáveis socioeconômicas e demográficas. O teste qui-quadrado foi usado para avaliar se a distribuição das proporções de (in)segurança alimentar foi estatisticamente diferente entre as categorias avaliadas. Considerou-se um p-valor $<0,05$. O software R 2.10.1 foi utilizado para a análise dos dados.

O projeto foi aprovado pela Secretaria de Trabalho, Assistência Social e Cidadania do município de Petrópolis e pelo Comitê de Ética da Faculdade de Medicina de Petrópolis, Faculdade Arthur Sá Earp Neto e Hospital Alcides Carneiro. A coleta de dados foi realizada após a assinatura do Termo de Consentimento Livre e Esclarecido pelo entrevistado, conforme estabelecido na Resolução CNS no 196/96.

\section{Resultados}

A tabela 1 mostra os resultados da distribuição dos domicílios de acordo com o grau de (in) segurança alimentar e fatores sociodemográficos. A prevalência de insegurança alimentar entre as famílias beneficiárias do restaurante popular (RP) foi de 42,9\%, dos quais 28,6\% apresentavam IA leve, 11,4\% tinham IA moderada e 2,9\% tinham IA grave. Já entre os domicílios incluídos no programa de abastecimento alimentar "Cesta Cheia, Família Feliz" (CCFF), 72,2\% tinham IA, sendo 48,9\% IA leve, 16,7\% moderada e 6,6\% grave.

Tabela 1. Situação de Segurança Alimentar e características socioeconômicas dos domicílios beneficiados pelo Restaurante Popular e pelo programa de distribuição de alimentos "Cesta Cheia, Família Feliz”, julho-outubro de 2009. Petrópolis, RJ.

\begin{tabular}{lcccc}
\hline \multirow{2}{*}{ Característica no Domicílio } & \multicolumn{2}{c}{$\begin{array}{c}\text { Restaurante } \\
\text { Popular }\end{array}$} & \multicolumn{2}{c}{$\begin{array}{c}\text { "Cesta Cheia } \\
\text { Família Feliz" }\end{array}$} \\
\cline { 2 - 5 } & $\mathrm{n}$ & $\%$ & $\mathrm{n}$ & $\%$ \\
\hline Situação de Segurança Alimentar & & & & \\
Segurança Alimentar & 60 & 57.1 & 25 & 27.8 \\
Insegurança Alimentar Leve & 30 & 28.6 & 44 & 48.9 \\
Insegurança Alimentar Moderada & 12 & 11.4 & 15 & 16.7 \\
Insegurança Alimentar Severa & 3 & 2.9 & 6 & 6.6
\end{tabular}


Classe socioeconômica $(A B E P)^{\#}$
A
2
B
12
1.9
60
11.5
1
1.1
C
57.7
44
50.6
D
29
27.9
38
43.7
E
1.0
4
4.6

Renda familiar mensal per capita (salário mínimo)*

$$
\begin{aligned}
& <1 / 4 \\
& 1 / 4-1 / 2 \\
& 1 / 2-1 \\
& 1+
\end{aligned}
$$$$
3
$$$$
24
$$$$
34
$$

Trabalho do chefe da família

Aposentado

Trabalha

Faz "biscate"

Não trabalha / desempregado

10

9.5

23

25.6

Grau de escolaridade do chefe da família

Analfabeto / até $3 \underline{a}$ série fundamental

Até $4^{\mathrm{a}}$ série fundamental

Fundamental completo

Médio completo

Superior completo

Participação em outros programas

Cesta básica do trabalho

8

7.6

"Programa Bolsa Família"

7

6.7

27

30.0

Outros

7

6.7

7

\#ABEP - Associação Brasileira de Empresas de Pesquisa; *Considerando o valor do salário mínimo no ano de $2009(\mathrm{R} \$ 465-\mathrm{US} \$ 245)$. 
As famílias incluídas no CCFF apresentaram condições socioeconômicas mais desfavoráveis, em comparação com os beneficiários do PR. O número de pessoas no domicílio variou entre uma

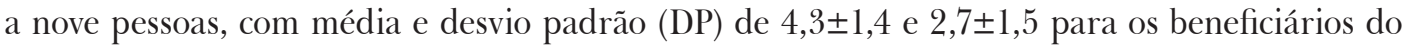
CCFF e RP, respectivamente. Cerca de $60 \%$ das famílias incluíam membros com menos de 20 anos de idade em ambos os programas (média e DP de 2,0 1,3 moradores nessa faixa etária por família para o CCFF e $0,6 \pm 0,9$ para o RP).

A classe econômica predominante em ambos os programas foi a classe $\mathrm{C}$ (representando $57,7 \% \mathrm{e}$ 50,6\% dos beneficiários do CCFF e do RP, respectivamente), seguido pela classe D (27,9\% e 43,7\%, respectivamente). Nenhuma família incluída no CCFF apresentou renda per capita superior a um salário mínimo por mês; em contraste, essa renda foi a mais comum entre os beneficiários do RP. No grupo de beneficiários do CCFF, aproximadamente $25 \%$ dos chefes de família não tiveram trabalho remunerado no mês anterior à entrevista ou estavam desempregados, em comparação com 9,5\% no RP, onde os aposentados representavam 46,7\% dos beneficiários.

O nível de escolaridade dos chefes das famílias atendidas pelos dois programas foi muito baixo, destacando-se um alto percentual de pessoas que estudaram até a $4^{a}$ - série fundamental $(65,5 \%$ e 52,9\% para CCFF e RP, respectivamente). Além disso, entre os beneficiários do CCFF, 30,0\% também recebiam auxílio de outro programa social do governo ("Programa Bolsa Família” - um programa de transferência de renda federal), em comparação com 6,7\% no RP.

$\mathrm{O}$ acesso ao abastecimento público de água foi relatado por $62,9 \%$ dos beneficiários do RP e 75,6\% do CCFF, destacando que 40,0\% e 30,0\% dos domicílios, respectivamente, não tinham tratamento de água potável. O sistema de coleta de esgoto estava presente em aproximadamente $80 \%$ dos domicílios. Constatou-se um amplo acesso à coleta de lixo, tanto no RP quanto no CCFF, abrangendo 98,1\% e 100\% dos domicílios, respectivamente.

Em relação às formas de obtenção de alimentos, a compra de alimentos em supermercados foi citada pela maioria dos beneficiários do RP $(94,3 \%)$ e por $90 \%$ dos beneficiários do CCFF. É importante ressaltar que os programas de fornecimento de alimentos foram a principal forma de obtenção de alimentos para as famílias incluídas no CCFF (93,3\%) e referida por 32,4\% dos beneficiários do RP.

As análises bivariadas utilizadas para investigar a relação entre IA e fatores sociodemográficos são demonstradas na tabela 2. A pobreza esteve relacionada à prevalência de IA. Domicílios classificados como pertencentes às classes D e E, e também com renda per capita inferior a um quarto do salário mínimo por mês tiveram a maior prevalência de IA. Também foi observada a influência do número de moradores e da presença de pessoas com menos de 20 anos de idade, presença de moradores menores de 6 anos no domicílio na situação de (in)segurança alimentar. Maiores prevalências de IA foram vistas em domicílios com quatro ou mais moradores (64,1\%), com membros com menos de 20 anos de idade (64,7\%) e em famílias com filhos menores de seis anos de idade (69,7\%). Também 
foi encontrada diferença estatisticamente significativa na prevalência de IA de acordo com a cor/ raça do chefe da família - 51,5\% entre brancos e 61,9\% entre pardos ou pretos.

Indicadores econômicos relacionados às condições de habitação também tiveram importante papel na situação de (in)segurança alimentar familiar. Aproximadamente $65 \%$ das famílias que não possuíam casa própria, 70\% que moravam em casas inacabadas e $75 \%$ que moravam em domicílios com apenas um cômodo vivenciavam situação de IA.

Não foi encontrada associação entre segurança alimentar e participação em outros programas governamentais, mas as famílias que recebiam algum benefício tenderam a relatar maiores níveis de IA, em comparação com aquelas não incluídas em outros programas.

Tabela 2. Prevalência de segurança alimentar de acordo com as variáveis sociodemográficas dos domicílios beneficiados pelo restaurante popular e pelo programa de distribuição de alimentos "Cesta Cheia, Família Feliz”, julho-outubro, 2009. Petrópolis, RJ.

\begin{tabular}{|c|c|c|c|c|c|}
\hline \multirow[b]{2}{*}{ Variáveis Sociodemográficas } & \multirow[b]{2}{*}{$\begin{array}{c}\text { Distribuição } \\
\text { da categoria } \\
(\%)\end{array}$} & \multirow[b]{2}{*}{$\begin{array}{c}\text { Segurança } \\
\text { Alimentar } \\
(\%)\end{array}$} & \multicolumn{2}{|c|}{ Insegurança Alimentar } & \multirow[b]{2}{*}{$P$-valor } \\
\hline & & & $\begin{array}{c}\text { Leve } \\
(\%)\end{array}$ & $\begin{array}{c}\text { Moderada } \\
\text { ou Severa } \\
(\%)\end{array}$ & \\
\hline \multicolumn{6}{|l|}{ Número de moradores no domicílio } \\
\hline $1-3$ & 52.8 & 50.5 & 29.1 & 20.4 & \multirow{2}{*}{0.026} \\
\hline $4+$ & 47.2 & 35.9 & 47.8 & 16.3 & \\
\hline \multicolumn{6}{|c|}{ Presença de moradores menores de 20 anos } \\
\hline Não & 40.5 & 55.7 & 25.3 & 19.0 & \multirow{2}{*}{0.007} \\
\hline Sim & 59.5 & 35.3 & 46.6 & 18.1 & \\
\hline \multicolumn{6}{|c|}{ Presença de moradores menores de 6 anos } \\
\hline Não & 71.3 & 48.9 & 30.9 & 20.1 & \multirow{2}{*}{0.006} \\
\hline Sim & 28.7 & 30.4 & 55.4 & 14.3 & \\
\hline \multicolumn{6}{|l|}{ Classe Socioeconômica (ABEP) ${ }^{\#}$} \\
\hline $\mathrm{D}-\mathrm{E}$ & 37.7 & 27.8 & 43.1 & 29.2 & \multirow{3}{*}{0.002} \\
\hline $\mathrm{C}$ & 54.5 & 48.1 & 39.4 & 12.5 & \\
\hline$A-B$ & 7.9 & 73.3 & 13.3 & 13.3 & \\
\hline \multicolumn{6}{|c|}{ Renda familiar mensal per capita (salário mínimo)* } \\
\hline $1+$ & 21.2 & 76.3 & 15.8 & 7.9 & \multirow{4}{*}{$<0,001$} \\
\hline $1 / 2-1$ & 25.1 & 48.9 & 37.8 & 13.3 & \\
\hline $1 / 4-1 / 2$ & 31.8 & 29.8 & 45.6 & 24.6 & \\
\hline$<1 / 4$ & 21.8 & 23.1 & 48.7 & 28.2 & \\
\hline
\end{tabular}


Cor/raça do chefe da família

\begin{tabular}{|c|c|c|c|c|c|}
\hline Branco & 50.0 & 48.5 & 40.2 & 11.3 & \multirow{2}{*}{0.033} \\
\hline Parda ou Preta & 50.0 & 38.1 & 36.1 & 25.8 & \\
\hline \multicolumn{6}{|l|}{ sse do domicílio } \\
\hline Próprio & 62.1 & 48.8 & 39.7 & 11.6 & \multirow{2}{*}{0.006} \\
\hline Não Próprio & 37.9 & 35.1 & 35.1 & 29.7 & \\
\hline \multicolumn{6}{|l|}{ onstrução da moradia } \\
\hline Alvenaria acabada & 63.1 & 51.2 & 33.3 & 15.4 & \multirow{2}{*}{0.019} \\
\hline Outros & 36.9 & 30.6 & 45.8 & 23.6 & \\
\hline \multicolumn{6}{|c|}{ úmero de cômodos (quartos e salas) } \\
\hline $3+$ & 55.4 & 53.7 & 34.3 & 12.0 & \multirow{3}{*}{$<0.001$} \\
\hline 2 & 32.3 & 33.3 & 47.6 & 19.0 & \\
\hline 1 & 12.3 & 25.0 & 29.2 & 45.8 & \\
\hline \multicolumn{6}{|c|}{ urticipação em outros programas } \\
\hline Sim & 25.6 & 34.0 & 42.0 & 24.0 & \multirow{2}{*}{ NS } \\
\hline Não & 74.4 & 46.9 & 36.6 & 16.6 & \\
\hline
\end{tabular}

\# ABEP - Associação Brasileira de Empresas de Pesquisa; * Considerando o valor do salário mínimo no ano de 2009 ( $\mathrm{R} \$ 465$ - US\$245).

\section{Discussão}

Aproximadamente $43 \%$ das famílias beneficiárias do restaurante popular (PR) e 72\% das famílias incluídas no programa de abastecimento alimentar (CCFF) foram classificadas em situação de insegurança alimentar. As condições socioeconômicas e de moradia foram mais desfavoráveis entre as famílias atendidas pelo CCFF - maior participação em outros programas sociais, renda e escolaridade baixas e, consequentemente, maior prevalência de classes econômicas mais baixas - poderiam explicar a diferença encontrada. Além disso, o programa CCFF auxilia famílias em condições precárias de vida previamente selecionadas a partir de um cadastro municipal, enquanto o RP, embora vise atender principalmente aos trabalhadores de baixa renda (formais e informais) e famílias em risco de IA, é acessível a toda a população. A prevalência de IA em ambos os grupos foi maior que a relatada pela Pesquisa Nacional por Amostra de Domicílios ${ }^{2}$ para o estado do Rio de Janeiro (28,3\%) e Brasil (34,8\%), presumivelmente porque o programa atrai famílias mais necessitadas.

Estudos realizados em outros países com populações vulneráveis à IA observaram prevalências semelhantes às do presente estudo. A prevalência de IA foi de $76 \%$ entre famílias de baixa e média renda com filhos matriculados em escolas primárias públicas de Bogotá, Colômbia, ${ }^{13} \mathrm{e}$ 
53,3\% em famílias com pelo menos um filho com menos de 12 anos de idade no Peru. ${ }^{14}$ MelgarQuinonez et al., medindo IA em áreas urbanas e rurais, relataram taxas de 70,3\% na Bolívia e 35,5\% nas Filipinas. ${ }^{15}$ Piaseu \& Mitchell encontraram prevalência de IA de 55,8\% em famílias de áreas carentes na Tailândia. ${ }^{16}$ Em estudo conduzido em comunidades rurais da Malásia, Shariff e Khor identificaram situação de IA em $58 \%$ das famílias de baixa renda. ${ }^{17} \mathrm{~A}$ alta prevalência de IA descrita acima apenas reforça que este é um problema complexo e de ampla abrangência.

Os fatores associados à IA incluíram nível econômico, renda familiar, posse do domicílio, tipo de construção da moradia e número de cômodos do domicílio. Estes e outros indicadores socioeconômicos também foram associados com a IA em outros estudos nacionais, ${ }^{18,19}$ bem como em estudos internacionais. ${ }^{13,20-23}$ Essas associações são esperadas; a relação com a renda e outros indicadores correlacionados é conceitual, já que o acesso à alimentação é o principal determinante da IA no Brasil. ${ }^{5}$ Além desses indicadores, o número de membros da família, a presença de moradores com menos de 20 anos, presença de moradores menores de 6 anos de idade e a cor/ raça do chefe da família também se associaram com a IA neste estudo e têm sido documentados em outras pesquisas. ${ }^{17-19,24,25}$

Mesmo em cidades onde o índice de desenvolvimento humano (IDH) é considerado muito alto, comparável a países desenvolvidos, como o município de Petrópolis $(\mathrm{IDH}=0,804 \mathrm{em}$ 2000), ${ }^{26}$ programas que assegurem o acesso à alimentação são essenciais. Essa necessidade se deve à desigualdade social, problema que assola, principalmente, os países em desenvolvimento. Infelizmente, apesar de uma queda no coeficiente de Gini no Brasil (de 0,64 em 1991 para 0,49 em 2009), ${ }^{27}$ ainda existe acentuada desigualdade e a renda continua muito concentrada, como consequência de distorções historicamente acumuladas. Em 2006, $10 \%$ da população com menor renda representaram apenas $1 \%$ da renda total do Brasil, enquanto 10\% dos trabalhadores com rendimentos mais elevados responderam por $44,4 \%$ da renda total. ${ }^{28}$

A garantia da segurança alimentar exige ações coordenadas que envolvam a articulação adequada entre as políticas estruturais - como distribuição de renda, geração de emprego, regulação da produção e distribuição de alimentos, reforma agrária, ampliação do acesso aos alimentos, entre outros - $\mathrm{e}$ as ações emergenciais denominadas políticas compensatórias. Também é essencial o controle da qualidade dos alimentos, o monitoramento do estado nutricional e da saúde das populações, além da promoção de hábitos alimentares saudáveis. Nesse contexto, a Política de Segurança Alimentar no Brasil pode ser considerada inovadora, uma vez que não propõe apenas medidas atenuantes em curto prazo, mas também visa mudanças estruturais, inclusão social e distribuição de renda para os mais pobres. Outros aspectos positivos são a inclusão do problema da fome como prioridade na agenda política brasileira e o fortalecimento da participação e mobilização da sociedade. ${ }^{29}$ 
Os critérios utilizados para a identificação da população vulnerável no programa "Fome Zero" apresentam alta sensibilidade em relação à pobreza e à fome - incluem indivíduos que ganham menos de um dólar por dia, linha internacional de pobreza estabelecida pelo Banco Mundial. ${ }^{6}$ Entretanto, a baixa especificidade na seleção da população-alvo, apesar de ampliar a cobertura do programa, dificulta a avaliação de seus resultados e impacto.

Entre 2001 e 2004, cinco milhões de brasileiros saíram da situação de pobreza e a redução na desigualdade de renda foi de $4 \%$. Em 2006, a renda média dos $50 \%$ mais pobres da população aumentou 11,99\%. Já a renda média dos $10 \%$ mais ricos aumentou 7,85\%. ${ }^{30}$ A diminuição da desigualdade é atribuída não somente ao crescimento econômico, ${ }^{31}$ mas também ao aumento na renda das famílias mais pobres, promovido pelo "Programa Fome Zero". ${ }^{32}$

Outros avanços relacionados à segurança alimentar no Brasil devem ser mencionados. Mais de 47 milhões de crianças são atendidas pelo Programa de Alimentação Escolar, uma das principais fontes de acesso à alimentação para famílias pobres. Outro programa a ser destacado é o Programa de Aquisição de Alimentos, que tem permitido a 118.000 agricultores familiares aumentar seus rendimentos. ${ }^{33}$ De uma maneira geral, os impactos de tais programas podem ser observados na melhoria significativa da saúde infantil no país - redução de $47 \%$ na taxa de mortalidade infantil entre 1990 a 2006 e redução na proporção de crianças menores de dois anos de idade com baixo peso de $12,7 \%$ em 2000 para $3,5 \%$ em $2006 .^{34}$

Por outro lado, a qualidade nutricional da alimentação está longe de ser ideal, um quesito importante do conceito de segurança alimentar. A diversidade dietética associa-se com a qualidade da alimentação, e tem sido relacionada a diversos desfechos positivos na saúde de indivíduos. ${ }^{35-37}$ Tendências sobre a disponibilidade de alimentos nos domicílios brasileiros nas últimas três décadas revelam que grupos de alimentos, incluindo carne, produtos lácteos, frutas e vegetais, são aqueles cuja participação na dieta aumenta de forma uniforme de acordo com o nível de renda familiar. ${ }^{38}$

Os programas que fornecem alimentos são criticados por não permitirem que as famílias escolham seus alimentos; portanto, é essencial avaliar se todo alimento que é fornecido é consumido. Deve-se destacar que ambos os programas contribuem para melhorar a qualidade da alimentação dos beneficiários. No RP, as refeições servidas são planejadas por nutricionistas e o CCFF distribui apenas produtos saudáveis - frutas, legumes e ovos. No entanto, incluir ações de educação nutricional promovendo a adoção de práticas alimentares saudáveis é fundamental. Ressalta-se que as iniciativas do governo, apoiadas por políticas públicas, devem ser avaliadas para possíveis ajustes. A investigação da prevalência de IA no grupo assistido representa um passo primordial nessa avaliação.

Algumas limitações devem ser mencionadas. Primeiro, o desenho seccional limita a capacidade de fazer inferências causais entre IA e as variáveis que podem mudar ao longo do tempo, como a 
participação em outros programas governamentais e a renda familiar mensal. Além disso, a falta de controle para possíveis fatores de confusão na análise apenas permite identificar diferenças na situação de segurança alimentar segundo variáveis socioeconômicas e demográficas.

\section{Conclusão}

Os resultados foram importantes para identificar as famílias mais vulneráveis que devem ser priorizadas em medidas assistenciais. Além disso, a alta prevalência de IA em determinados grupos socioeconômicos pode confirmar a desigualdade que permanece no município e demonstrar que os programas estão atingindo de forma adequada o grupo alvo. Em curto prazo, o acesso aos alimentos em comunidades de baixa renda é garantido.

Garantir a segurança alimentar não é uma tarefa fácil, sobretudo em um país historicamente marcado pela desigualdade social como o Brasil. O primeiro passo foi dado, mas ainda resta muito a ser feito. Ferramentas simples, como a EBIA, podem ser utilizadas para monitorar a desigualdade social e também ajudar os governos a combatê-la.

Os próximos passos incluem esforços para entender melhor o impacto dos programas sociais na segurança alimentar das famílias. Também é fundamental explorar o acesso a alimentos de qualidade com preços acessíveis entre as famílias incluídas nos programas de combate a pobreza e IA.

\section{Referências}

1. Conselho Nacional de Segurança Alimentar e Nutricional (Brasil). Princípios e diretrizes de uma Política de Segurança Alimentar e Nutricional. Textos de Referência da II Conferência Nacional de Segurança Alimentar e Nutricional. Brasília: CONSEA; 2004.

2. IBGE. Pesquisa Nacional por Amostra de Domicílios (PNAD) 2004. Segurança Alimentar. Rio de Janeiro: IBGE; 2006.

3. Food and Agriculture Organization of the United Nations. Food Security Statistics. FAO; 2009. Disponível em: http://www.fao.org/economic/ess/food-security-statistics/en/

4. Belik W, Silva JG, Takagi M. Políticas de combate à fome no Brasil. São Paulo Perspec. 2001;15:119-29.

5. Maluf RS, Menezes F, Valente FL. Contribuição ao tema da Segurança Alimentar no Brasil. Cadernos de Debate. 1996;4:66-88.

6. Vasconcelos FAG. Combate à fome no Brasil: uma análise histórica de Vargas a Lula. Rev Nutr. 2005;18:439-57.

7. Yasbek MC. O programa Fome Zero no contexto das políticas sociais brasileiras. São Paulo Perspec. 2004;18:104-12. 
8. Costa C, Maluf RS. Diretrizes para uma Política Municipal de Segurança Alimentar e Nutricional. São Paulo: Pólis; 2001.

9. Maluf RS. Ações públicas locais de abastecimento alimentar. São Paulo: Pólis; 1999. Pólis Papers n. 5.

10. Bickel G, Nord M, Price C, Hamilton W, Cook J. Guide to Measuring Household Food Security. Alexandria, VA: USDA; 2000.

11. Perez-Escamilla R, Segall-Correa AM, Maranha LK, Sampaio MFA, Marín-León L, Panigassi G. An adapted version of the U.S. Department of Agriculture Food Insecurity Module is a valid tool for assessing household Food Insecurity in Campinas, Brazil. Journal of Nutrition. 2004;134:1923-8.

12. Associação Brasileira de Empresas de Pesquisa. Dados com base no Levantamento Sócio Econômico 2008 - IBOPE; 2010. Disponível em: http://www.abep.org/

13. Isanaka S, Mora-Plazas M, Lopez-Arana S, Baylin A, Villamor E. Food Insecurity is highly prevalent and predicts underweight but not overweight in adults and school children from Bogota, Colombia. Journal of Nutrition. 2007;137(12):2747-55.

14. Vargas S, Penny ME. Measuring food insecurity and hunger in Peru: a qualitative and quantitative analysis of an adapted version of the USDA's Food Insecurity and Hunger Module. Public Health Nutr. 2010;13(10):1488-97.

15. Melgar-Quinonez HR, Zubieta AC, MkNelly B, Nteziyaremye A, Gerardo MF, Dunford C. Household food insecurity and food expenditure in Bolivia, Burkina Faso, And the Philippines. Journal of Nutrition. 2006;136(5):S1431-7.

16. Piaseu N, Mitchell P. Household food insecurity among urban poor in Thailand. J Nurs Sch. 2004;36(2):115-21.

17. Shariff ZM, Khor GL. Household food insecurity and coping strategies in a poor rural community in Malaysia. Nutr Res Pract. 2008;2(1):26-34.

18. Salles-Costa R, Pereira RA, Vasconcellos MTL, Veiga GV, Marins VMR, Jardim BC et al. Association between socioeconomic factors and food insecurity: a population-based study in the Rio de Janeiro metropolitan area, Brazil. Rev Nutr. 2008;21:S99-109.

19. Panigassi G, Segall-Corrêa AM, Marin-León L, Pérez-Escamilla R, Sampaio MFA, Maranha LK. Food insecurity as an indicator of inequity: analysis of a population survey. Cad Saúde Pública. 2008;24:2376-84.

20. Gulliford MC, Nunes C, Rocke B. The 18 Household Food Security Survey items provide valid food security classifications for adults and children in the Caribbean. BMC Public Health. 2006;6:26.

21. Foley W, Ward PR, Carter P, Coveney JD, Tsourtos G, Taylor A. An ecological analysis of factors associated with food insecurity in South Australia, 2002-7. Public Health Nutr. 2010;13(2):215-21.

22. Oh SY, Hong MJ. Food insecurity is associated with dietary intake and body size of Korean children from low-income families in urban areas. Eur J Clin Nutr. 2003;57(12):1598-1604.

23. Gulliford MC, Mahabir D, Rocke B. Food insecurity, food choices, and body mass index in adults: nutrition transition in Trinidad and Tobago. Int J Epidemiol. 2003;32(4):508-16. 
24. Furness BW, Simon PA, Wold CM. Asarian-Anderson, J. Prevalence and predictors of food insecurity among low-income households in Los Angeles County. Public Health Nutr. 2004;7(06):791-4.

25. Kaiser L., Baumrind N, Dumbauld S. Who is food-insecure in California? Findings from the California Women's Health Survey, 2004. Public Health Nutr. 2007;10(06):574-81.

26. Programa das Nações Unidas para o Desenvolvimento. Desenvolvimento Humano e IDH. Brasília: PNUD; 2010. Disponível em: http://www.pnud.org.br/atlas/

27. IPEA. Desigualdade e pobreza no Brasil metropolitano durante a crise internacional: primeiros resultados. Brasília: IPEA; 2009.

28. Barros RP, Carvalho M, Franco S, Mendonça R. A queda recente da desigualdade de renda no Brasil. Rio de Janeiro: IPEA; 2007. Texto para Discussão n. 1258.

29. Belik W, Del Grossi M. O Programa Fome Zero no contexto das políticas sociais no Brasil. Cadernos de Debate. 2003;X:1-24.

30. Neri M. O real do Lula. Conjuntura Econômica. 2007;61(10):42-5.

31. IPEA. Nota técnica sobre a recente queda da desigualdade de renda no Brasil. Brasília: IPEA; 2006.

32. Jaccoud L. Indigência e pobreza: efeitos dos benefícios previdenciários, assistenciais e de transferências de renda. In: Peliano AM (editor). Desafios e Perspectivas da Política Social. Brasília: IPEA; 2006. Texto para Discussão n. 1248.

33. Rocha C. The Positive Experience of Brazil in Combating Hunger. Presentation to the Parliamentary Meeting on the Occasion of the World Summit on Food Security. Rome; 2009.

34. United Nations Children's Fund. Progress for Children: A World Fit for Children. Statistical Review no. 6. New York: UNICEF; 2007.

35. Hoddinott J, Yohannes Y. Dietary diversity as household food security indicator. Washington, D.C.: International Food Policy Research Institute; 2002. Discussion Paper n. 136.

36. Kant AK. Indexes of Overall Diet Quality: A Review. J Am Diet Assoc. 1996;96(8):785-91.

37. Savy M, Martin-Prevel Y, Sawadogo P, Kameli Y, Delpeuch F. Use of variety/diversity scores for diet quality measurement: relation with nutritional status of women in a rural area in Burkina Faso. Eur J Clin Nutr. 2005;59(5):703-16.

38. Levy-Costa RB, Sichieri R, Pontes NS, Monteiro CA. Household food availability in Brazil: distribution and trends (1974-2003). Rev Saúde Pública. 2005;39(4):530-40.

Recebido: 01/7/2013

Aprovado: 30/8/2013 